\title{
Psychiatric morbidity in Parkinson's Disease: a case report
}

\author{
Amitabh Saha \\ Department of Psychiatry, Srinagar, Jammu \& Kashmir, India
}

Email address:

sahaing@gmail.com (A. Saha)

\section{To cite this article:}

Amitabh Saha. Psychiatric Morbidity in Parkinson's Disease: A Case Report, American Journal of Life Sciences. Vol. 1, No. 2, 2013 , pp. 27-30. doi: 10.11648/j.ajls.20130102.11

\begin{abstract}
Psychiatric disorders like anxiety and depression are common in patients with Parkinson's disease. Patients are needed to be managed on a comprehensive basis, in which he can receive psychiatric management along with his medical advice and prescription. Parkinson's disease is an illness affecting the geriatric age group resulting in debilitating and disfiguring disease associated with the presence of resting tremor, bradykinesia, and postural instability. The prevalence of social anxiety disorder in these patients is important, because further determines the improvement of the patient and his response to drugs.
\end{abstract}

Keywords: Parkinson's Disease, Social Phobia, Anxiety Disorder, Debilitating Disorder, Depression, Bradykinesia, Antiparkinson's Medications

\section{Introduction}

Parkinson's disease is a degenerative disorder of the central nervous system. The motor symptoms of Parkinson's disease result from the death of dopaminegenerating cells in the substantia nigra, a region of the midbrain; the cause of this cell death is unknown. Early in the course of the disease, the most obvious symptoms are movement-related; these include shaking, rigidity, slowness of movement and difficulty with walking and gait. Later, cognitive and behavioural problems may arise, with dementia commonly occurring in the advanced stages of the disease. Other symptoms include sensory, sleep and emotional problems. Parkinson's disease is more common in the elderly, with most cases occurring after the age of 50 .

The main motor symptoms are collectively called parkinsonism, or a "parkinsonian syndrome". Parkinson's disease is often defined as a parkinsonian syndrome that is idiopathic (having no known cause), although some atypical cases have a genetic origin. Parkinson's disease affects movement, producing motor symptoms. Non-motor symptoms, which include autonomic dysfunction, neuropsychiatric problems (mood, cognition, behavior or thought alterations), and sensory and sleep difficulties, are also common. Some of these non-motor symptoms are often present at the time of diagnosis and can precede motor symptoms. Four motor symptoms are considered cardinal in Parkinson's disease : tremor, rigidity, slowness of movement, and postural instability. Tremor is the most apparent and well-known symptom. It is the most common; though around $30 \%$ of individuals with Parkinson's disease do not have tremor at disease onset, most develop it as the disease progresses. It is usually a rest tremor: maximal when the limb is at rest and disappearing with voluntary movement and sleep.

Bradykinesia (slowness of movement) is another characteristic feature of Parkinson's disease, and is associated with difficulties along the whole course of the movement process, from planning to initiation and finally execution of a movement. Performance of sequential and simultaneous movement is hindered. Bradykinesia is commonly a very disabling symptom in the early stages of the disease. Bradykinesia is not equal for all movements or times. It is modified by the activity or emotional state of the subject, to the point that some patients are barely able to walk yet can still ride a bicycle. Generally patients have less difficulty when some sort of external cue is provided.

Rigidity is stiffness and resistance to limb movement caused by increased muscle tone, an excessive and continuous contraction of muscles. In parkinsonism the rigidity can be uniform (lead-pipe rigidity) or ratchety (cogwheel rigidity). The combination of tremor and increased tone is considered to be at the origin of cogwheel rigidity. Rigidity may be associated with joint pain; such 
pain being a frequent initial manifestation of the disease. In early stages of Parkinson's disease, rigidity is often asymmetrical and it tends to affect the neck and shoulder muscles prior to the muscles of the face and extremities. With the progression of the disease, rigidity typically affects the whole body and reduces the ability to move.

Postural instability is typical in the late stages of the disease, leading to impaired balance and frequent falls, and secondarily to bone fractures. Instability is often absent in the initial stages, especially in younger people

Parkinson's disease can cause neuropsychiatric disturbances which can range from mild to severe. This includes disorders of speech, cognition, mood, behaviour, and thought.

Cognitive disturbances can occur in the initial stages of the disease and sometimes prior to diagnosis, and increase in prevalence with duration of the disease. The most common cognitive deficit in affected individuals is executive dysfunction, which can include problems with planning, cognitive flexibility, abstract thinking, rule acquisition, initiating appropriate actions and inhibiting inappropriate actions, and selecting relevant sensory information. Fluctuations in attention and slowed cognitive speed are among other cognitive difficulties. Memory is affected, specifically in recalling learned information. Nevertheless, improvement appears when recall is aided by cues.

A person with Parkinson's disease has two to six times the risk of suffering dementia compared to the general population. The prevalence of dementia increases with duration of the disease. Dementia is associated with a reduced quality of life in people with Parkinson's disease and their caregivers, increased mortality, and a higher probability of needing nursing home care.

Behavior and mood alterations are more common in Parkinson's disease without cognitive impairment than in the general population, and are usually present in Parkinson's disease with dementia. The most frequent mood difficulties are depression, apathy and anxiety. Impulse control behaviors such as medication overuse and craving, binge eating, hypersexuality, or pathological gambling can appear in Parkinson's disease and have been related to the medications used to manage the disease. Psychotic symptoms - hallucinations or delusions-occur in $4 \%$ of patients, and it is assumed that the main precipitant of psychotic phenomena in Parkinson's disease is dopaminergic excess secondary to treatment; it therefore becomes more common with increasing age and levodopa intake.

Parkinson's disease can impair other body functions. Sleep problems are a feature of the disease and can be worsened by medications. Symptoms can manifest as daytime drowsiness, disturbances in REM sleep, or insomnia.

Parkinson's Disease is associated with various psychiatric disabilities like depression, anxiety, somatoform disorders, cognitive impairment like dementia and delirium in advanced stages(1-3).Parkinson's disease has both medical and a psychiatric morbidity. Social phobia is an irrational and intense fear of one or more social situations. Most sufferers fear only specific social situations, although those with generalized social phobia fear most social encounters.

In Parkinson's disease the patient may develop features of anxiety disorder in form of having avoidance response, persistent fear, inability to address other people or maintain adequate interpersonal relationships leading to social decline. Social anxiety was diagnosed in $16 \%$ of patients with Parkinson's disease.

Depression in Parkinson's disease is common, and its impact can equal that of the motor symptoms. The prevalence rates vary significantly, reflecting study design and the lack of uniform criteria for diagnosing depression. Depression, if undiagnosed or unacknowledged, can wreak havoc upon efforts to control motor symptoms, and may create major family and spousal tensions at a time when a patient most needs support. Depression may precede the diagnosis of Parkinson's disease, sometimes by years, and may need treatment before treatment of the parkinsonian motor symptoms is necessary. Patients with a prior history of depression are more vulnerable to developing depression with their Parkinson's disease. Depression may also compromise intellectual functions, giving an erroneous impression of dementia, a presentation known as pseudodementia. In the Parkinson's disease patient, demoralization at disability, loss of autonomy, the prospect of a chronic progressive neurological disorder, the need to take daily medication with no end in sight, and the truncation or abandonment of life goals and long-term plans may be the primary disturbance behind the patient's sadness.

Anxiety is frequently a major component of depression and is often disproportionate to parkinsonian disability. Among the various Anxiety disorders known, Social anxiety disorder,Generalized Anxiety disorder, Mixed anxiety and Depressive disorder are commonly seen. Common in Parkinson's disease, anxiety manifests itself as constant or episodic feelings of fear, incessant, uncontrollable worrying, and a variety of physical symptoms including muscle tension, palpitations, sweating, and shortness of breath. Treatment depends upon correctly identifying the source of anxiety. Anxiety is frequently part of the presenting symptoms of a depressive illness, and at times may be the dominant symptom. Anxiety often accompanies the "off" state - the periods of parkinsonian immobility. The anxiety associated with the off state can be severe.

Psychosis in Parkinsons disease is also well known. About $20 \%$ of patients with Parkinson's disease who receive long-term treatment with dopaminergic agents (e.g., levodopa, bromocriptine, pergolide ropinerole, and pramipexole) will develop psychosis identified by the presence of chronic hallucinations and delusions. 


\section{Case Report}

A 62 year old person, married, teacher by profession had been in the teaching profession for last 22 years. He gradually started noticing that he would have tremors in his fingers of his right hand. He initially took no notice of it but as he would write on the blackboard or hand over items to other people his fingers would show obvious tremors. He noticed that his handwriting had changed to an extent to which even his students started saying as to what exactly he had written on the board. Over the next few months he noticed that he had become more sluggish and lethargic and developed sleep disturbance. When his family members noticed that he had even stopped his morning walks, which he would religiously follow earlier, and that increased letharginess was leading to a decline in his work output that they became worried. He slowly developed pain in the neck and shoulders which later was seen over the smaller joints of his hands. When he was shown to the family physician he advised him some rest with some vitamin supplements. However when he would start to take the class he would develop a foreboding apprehension that he had forgotten what he had to teach. He was a Physics teacher and his drawings and writings on the board had significantly become ineligible and he became acutely aware of it when his students started to question his markings and writings. At home he noticed that his finger tremors had now increased to a large extent and that his handwriting skills had reduced in that he was writing much smaller than before. His spouse noticed a marked difference in his day to day activities in that there was marked slowness of his movements and that his joint pains had increased in intensity. He had no past history of any mental or neurological illness.

A Neurophysician, who on clinical examination, found features of pill rolling tremors, bradykinesia, cogwheel rigidity which patient had earlier felt as joint pains. The patient was advised routine hematological and biochemical tests with CT Scan and later MRI Scan of the brain. He was placed on combination treatment with levadopa and carbidopa (which is a peripheral dopa decarboxylase inhibitor). Patient showed some improvement in his symptoms but he noticed that he whenever he would enter the classroom to teach he would develop feelings of apprehension, fearfulness, mounting anxiety, palpitations, feeling that he was being judged and ridiculed by the students because of his ailment. He developed fear of public speaking and initially would try to avoid teaching anything new in the class room and would leave the class midway as he did not want to be the centre of attention. As his occupational profile started to suffer and more complaints mounted against him he took a break from school to undergo complete treatment.

After two months of treatment when he resumed teaching his class he noticed an insurmountable anxiety that he would do something that would be unacceptable to societal norms, developed feelings of giddiness and fear that he was not able to breathe properly. His symptoms only aggravated further and he found himself avoiding public discourse, lectures or any condition that entailed him to be the centre of attention. He started avoiding taking classes and later stopped going to school to teach altogether to prevent people from seeing his disabilities.

He became acutely aware of his slow gait, poor concentration, stiffness of body limbs but what made it worse was the anticipatory anxiety he would perceive when he was made the focus of attention of any group, party or class. He was advised psychiatric referral by his neurologist. The patient was evaluated in detail by the psychiatrist who diagnosed him with having Social Phobic anxiety disorder as he had the characteristic features of avoidance response, persistent fear, and inability to address other people or maintain adequate interpersonal relationships leading to socio- occupational decline. $\mathrm{He}$ was placed on Tab Sertraline $50 \mathrm{mg}$ OD, Tab Inderal $40 \mathrm{mg}$ BD, Tab Clonazepam $1 \mathrm{mg}$ TDS initially which was later tapered down to $1 \mathrm{mg}$ HS. The dose of Tab Sertraline was slowly raised to therapeutic dose of $150 \mathrm{mg}$ OD.

At the end of 6 weeks of therapy, patient showed subjective improvement in his symptomatology of apprehension, sleep disturbance and anxiety. After 06 months the symptoms of Avoidance response also reduced to a large extent. The care givers were satisfied with the combination treatment given for both Parkinson's disease and social phobia as the patient had resumed his societal role as a teacher and he started taking classes for his students without any problem.

\section{Discussion}

Psychiatric disorders like anxiety and depression are common in patients with Parkinson's disease .Patients with disfiguring diseases experience significant distress in social interaction, and they may also develop social anxiety either related to or secondary to Parkinson's disease. Previous studies have highlighted the presence of social anxiety related to conditions like acne, psoriasis, hyperkinesias, obesity, and stuttering (4-13).

An extensive literature survey yielded references of Parkinson's disease associated with social anxiety disorder. Social Phobia is distinguished by a fear of public humiliation or embarrassment. It is one of several phobia disorders, which are all typified by excessive, specific, and consistent fear and avoidance of an object, activity, or situation. People with social phobia may avoid doing activities in public such as eating or speaking, as well as using public bathrooms. Ultimately, people with associated co-morbidities like a medical illness like Parkinson's disease with social phobia, fear that people may judge them, which would cause them to have mounting anxiety.

However, in DSM-IV, social anxiety related to a general medical condition like stuttering or trembling in Parkinson's disease is excluded from the diagnosis of social anxiety disorder and these patients receive the diagnosis of 
"Anxiety Disorder Not Otherwise Specified "(14). Such a procedure has been criticized for limiting the diagnosis of this condition $(7,11)$.

Two studies did report high frequencies of social anxiety in patients with Parkinson's disease. Stein et al (15) reported in a small sample $(\mathrm{N}=16)$ that $29 \%$ of the patients had social anxiety. In the second study, the frequency of social phobia was $11.5 \%$ in a large sample of patients $(\mathrm{N}=384)$ with Parkinson's disease (16)

In another study, none of the patients with Parkinson's disease had social anxiety in a comparative study investigating psychiatric morbidity in patients with cervical dystonia and Parkinson's disease (17). Since secondary social anxiety in patients with Parkinson's disease had not been investigated in previous studies, this present study aimed to examine the association between social anxiety related to Parkinson's disease in a patient. In this case report, there appears to be an association between the development of a social anxiety disorder in patients suffering from Parkinson's disease.

Limitations of study: More studies will however be needed to see the type and severity of psychiatric illnesses with the disease progression in Parkinson's disease. A neurological workup with prognostic determinates is important to charter accompanying psychiatric illness. Primary or secondary nature of any anxiety disorder was not assessed in these studies. In other words, these studies did not differentiate whether social anxiety was secondary or related to Parkinson's disease or not.

Conflict of interest- none

\section{References}

[1] Reijnders JS, Ehrt U, Weber WE, et al: A systematic review of prevalence studies of depression in Parkinson's disease. Mov Disord 2008; 23:183-189.

[2] Lauterbach EC: The neuropsychiatry of Parkinson's disease and related disorders. Psychiatr Clin North Am 2004; $27: 801-825$

[3] Nuti A, Ceravolo R, Piccinni A, et al: Psychiatric comorbidity in a population of Parkinson's disease patients. Eur J Neurol 2004; 11:315-320.

[4] Oberlander EL, Schneier FR, Liebowitz MR: Physical disability and social phobia. J Clin Psychopharmacol 1994; $14: 136-143$

[5] Stein MB, Baird A, Walker JR: Social phobia in adults with stuttering. Am J Psychiatry 1996; 153:278—280.

[6] Devrimci-Özgüven H, Kudakçi N, Boyvat A: Secondary social anxiety in patients with psoriasis. Turk Psikiyatri Derg 2000; 11:121-126.

[7] Gündel H, Wolf A, Xidara V, et al: Social phobia in spasmodic torticollis. J Neurol Neurosurg Psychiatry 2001; $71: 499-504$

[8] Schneier FR, Barnes LF, Albert SM, et al: Characteristics of social phobia among persons with essential tremor. J Clin Psychiatry 2001; 62:367-372.

[9] Rosik CH: Psychiatric symptoms among prospective bariatric surgery patients: rates of prevalence and their relation to social desirability, pursuit of surgery, and followup attendance. Obes Surg 2005; 15:677—683.

[10] Topcuoglu V, Bez Y, Sahin Bicer D, et al: Social phobia in essential tremor. Turk Psikiyatri Derg 2006; 17:93-100.

[11] Ozel-Kizil ET, Akbostanci MC, Ozguven HD, et al: Secondary social anxiety in hyperkinesias. Mov Disord 2008; $23: 641-645$.

[12] Yolaç Yarpuz A, Demirci Saadet E, Erdi Sanli H, et al: Social anxiety in acne vulgaris patients and relationship with clinical variables. Turk Psikiyatri Derg 2008; 19:29_ 37.

[13] Ozel-Kizil ET: Social anxiety secondary to a general medical condition, in Social Phobia: Etiology, Diagnosis, and Treatment. Edited by Axelby CP. New York, Nova Publishers, 2009, pp 261-268.

[14] American Psychiatric Association: Diagnostic and Statistical Manual of Mental Disorders, 4th ed. Washington, DC, American Psychiatric Association, 1994.

[15] Stein MB, Heuser IJ, Juncos JL, et al: Anxiety disorders in patients with Parkinson's disease. Am J Psychiatry 1990; 147:217-220.

[16] de Rijk C, Bijl RV: Prevalence of mental disorders in persons with Parkinson's disease. Ned Tijdschr Geneeskd $1998 ; 142: 27-31$.

[17] Lauterbach EC, Freeman A, Vogel RL: Differential DSM-III psychiatric disorder prevalence profiles in dystonia and Parkinson's disease. J Neuropsychiatry Clin Neurosci 2004; $16: 29-36$. 